\title{
Music and Yoga - Perfect Pairing
}

\author{
Dr. Uma Vijay \\ Assistant Professor, Department of Music, Maheshwari College, Jaipur
}

\begin{abstract}
The objective of this study is to highlight the therapeutic effects of music on yoga and to provide a comprehensive review of the benefits of regular yoga practice along with soothing music. The participation rates in mind-body fitness Yoga programs is continuously increasing and thus it is important to be informed about the effects of Yoga and Music together. It is a well experienced fact that music has the ability to regulate a wide range of both positive and negative emotions. Thus, this manuscript explains the degree of music's influence on Yoga and since both focus on enhancing human performance and promote beneficial psychological state, the pairing of the two therapies is highlighted. It is concluded that yoga/meditation with music will enhance the states of flow and mindful awareness and reduce confusion.
\end{abstract}

Key words : Yoga, Music, Meditation.

\section{Pairing Music and Yoga}

Music is the universal language of the soul. It has the ability to dig into the deepest corners of our minds and take us to another time and place. Music and yoga have healing powers and are both considered as parts of alternative medicine. Thus pairing Music and Yoga together can create limitless possibilities of healing and transformation. Although Yoga can be practiced in silence, its effects can be magnified if it is paired with nice and pleasant Music.

\section{Mind Control using Rhythmic Sound}

Sound is capable of producing powerful reactions in the listener and this is the reason why our ears and emotions have such a strong bond. Pleasant sounds have a therapeutic effect on the body. It soothes all our bodily systems and activates the body's natural healing process. The vibration of the sound calms the nervous system and a profound sense of peace is obtained. It also de-stresses and facilitates better concentration and memory power. It is essential for all of us to have a sound body and calm mind. Once we are free from all worries and have achieved stability in mind and body, our lives will become bliss.

Our mind easily becomes absorbed in sound and so we enjoy listening to music and eventually feel inner bliss. With practice, the mind becomes calm and transparent and it starts understanding the Aahat as well as the Anahat Sound. The immense power of sounds and vibrations are used to improve mental health, bringing a more positive state of mind, which further helps in enhancing the quality of life. These sounds and vibrations create divine levels of energy and healing environment resulting in complete 
shift in the state of consciousness. A person can move to a deeperstate, the communication between mind, body and spirit is enhanced, resulting in ultimate bliss.

\section{Enhancing Yoga Experience with Music}

Music is preferred to be played during Yoga and Meditation simply on account of its positive effects and how it has proven to accelerate the healing process and facilitate movement and flow of body during exercise. Even scientific studies validate the positive benefits of calm, soothing music on the mind and body. Music and yoga both provide healing and therapy for undefined and most often unseen ailments. So it stands to reason that bringing these two therapies together has the potential for creating a power packed experience.

Music stimulates Chakras. Music is found to have profound effects on the subtle Energy Centres in our body called Chakras. According to Ayurveda Science, the health of an individual can be determined by measuring the state of these Seven Chakras in a Human body. Each of these Chakras has a sound frequency associated with it, and these sounds can be used for Chakra balancing and healing.

Music boosts endurance levels. Music can have a powerful effect on mood that it can help boost endurance levels during Yoga.

Music Elevates. Music helps in calming people down, improving levels of concentration, easing stress and elevating to a higher level of consciousness.

Music calms our emotions. Music soothens our emotions and helps meditate better and produces positive energy and aids in enhancing our overall health.

Music trains the mind. The mind has a tendency to wander, worry and drown into anxieties, fears and apprehensions. The only way to reduce the unwanted thoughts is by putting all the sense organs to one point to some extent. This state can be easily achieved with Music during Yoga practice since Yoga and Meditation helps to reach state of tranquility and music helps withdrawal from all the sense organs to reach this state and calm the unstable, highly spirited and impulsive mind.

Music changes mood. Music when played during yoga helps people to move from one level of spiritual consciousness to another with ease since it has the ability to change a person's mood in a few seconds.

Music reduces unwanted thoughts. During Yoga, Music plays a major role to reduce unwanted thoughts and focus attention to get good concentration in practice.

Music improves meditation. The moment we close our eyes to meditate, our minds tend to become more active and this paves the way to both positive and negative thoughts. Here Music helps since it calms our volatile thoughts and allows us to find stillness in the mind and acquire State of Tranquility.

Music creates a positive mood. We instantly get lifted out of a bad mood just by listening to a favourite song. Our brain's electrical signals are strengthened or weakened depending on our mood which can be altered through music which in turn influences our state of mind and health in general. 
Music enhances movement. Listening to music while doing Yoga eases our physical discomfort and Yoga becomes pleasure. The Transition from one pose to the next becomes effortless since the Music by acting as a Motivator, prevents us from focusing on specific physical sensations of fatigue. Music thus alters the mind's arousal state and also helps in gearing up the person for an increased exercise performance. When Music is introduced to Yoga routine, it allows the individual to continue to exercise with a greater efficiency wherein individuals can increase workload or time to exhaustion.

Music enhances effect of Yoga. We know that what we hear unconsciously also we take it to our hearts but the act of consciously listening or giving ear to Music during Yoga Practice constitutes a deliberate external act and this act using our sensory organs affects us positively. This way positive sound enhance the effect of Yoga \& Meditation.

Thus Music when paired with Yoga becomes a Sound Healing Technique in which sounds, vibrations, musical tones and frequencies are used to help restore, maintain, and improve one's state of mind, body and spirit. Soft and tranquil music playing in the background is most appropriate while doing Yoga and Meditation which strengthens us with courage and optimism. With a deeper understanding of music and its dimensions, one can harness the healing power of sound very effectively, successfully, scientifically and spiritually.

\section{Playing the Right Kind of Music during Yoga and Meditation}

Soft, soothing and meditative sounds are also needed to combat the noise emerging from chaotic environment. To understand the perfect type of music to be played during Yoga and Meditation we must silently observe the remarkable difference in the sounds of birds, animals, insects and other natural noises during the cycles of day and these sounds change with the seasons as well. Even the pitch of our own voice changes as the day goes by and we have to synchronise with the pattern of sound present at particular time of the day. Each hour of the day represents a different mood and a different state of being. Listening to music which is synchronised to the sympathetic overtones of the season, time and hour of the day makes us flow in harmony with the natural environment, saves energy, regulates moods and provides inspiration to live. Indian Classical Music is rich in this field and Ragas are created by Great Musicians who heard the sounds of nature at seasonal changes and during the cycle of the day and then created these Raagas to suit moods at various parts of the day and seasons. These Musicians composed with the consciousness of the harmonics of the time and hour of the day. Thus the music to be played during Yoga practice should be harmonious with these changes.

Music for morning meditation should produce a very tender, calming and refreshing meditative mood that helps us step out of sleep and into a harmonious waking state. If such music is heard continuously then it serves as an healing agent and increases personal magnetism. Music for evening meditation should inspire feelings of joy, confidence, satisfaction, inspiration and the calmness of moonlight. Listening to such kind of music improves mental and physical health and relieves stress. The droning 
sound of Tanpura at the backdrop subtly influences the nervous system. Such kind of music produces a meditative mood and brings improved mental and physical health.

We must be aware that not all types of music are ideal for use in Yoga. There are several music elements such as the beat, tune, and instrument used that enables to facilitate in the meditative aspect of Yoga. Indian music has its own distinct, enticing quality and also has a religious origin. They are absolutely wonderful for relaxing and meditating since they are specifically geared towards aiding meditation. Instrumental Classical Music or OM Chanting can be used for more rigorous and intensive yoga exercises and meditation. The instrumental music being devoid of words or lyrics make it more appropriate for yoga practice and meditation since it will not distract an individual's attention away from the practice and hence will provide a boost of energy to keep going. The instruments used in such music generate tones in perfect harmonic scale to induce calmness and create a meditative mood.

Today we can find a wide range of Music specifically intended to enhance Yoga and Meditation Practice, composed by experts mostly based on Indian Classical Music with roots in spirituality especially designed to enhance meditation practice. Choosing the right kind of music for Yoga is necessary since not all types of music harbour the same relaxing benefits. Some music, particularly the loud ones, can be very distracting and potentially harmful. Few suggestions are:

The Sound of Nature: We can find albums where sounds of the natural world like the birds singing, river flowing, the sound of the waves, etc. are recorded and are combined with music to be used as a therapeutic tool for stress relief and meditation.

Chanting of Mantras: Rhythmic Mantra Chanting along with traditional Indian instruments such as Tabla, Bansuri, Santoor, Sitar etc. can be used while doing Yoga.

Instrumental Classical Music: Instrumental Music provides a soothing melody to help soothen the minds. Indian Classical music offers a wide range of options of instruments like Bansuri, Santoor, Sitar, Sarod etc. which can be used according to the interest and taste.

Music balances the emotions and moods of human beings through its rhythmic pattern of tones and when paired with Yoga, can provide effective healing therapy for even the unseen and unexplained illnesses. Thus Bringing these two therapies together could definitely create a powerful and fulfilling experience.

\section{References}

Sharma, P.D. (1995). Yoga-Yogasana and Pranayama for Health, Navneet Publications. Swami Ramdev (2003). Yog Sadhana va Yog Chikitsa Rahasya, Divya Prakashan.

Swami Ramdev (2009). Pranayama Rahasya, Divya Prakashan.

www.lexiyoga.com/music-and-yoga

www.musicalyogaadventures.com

www.healthandyoga.com

www.doyouyoga.com/yoga-music

www.livestrong.com 